period $2(n=113)$ so we calculated three inadequate seizure ratings (expressed as a percentage of the total number of first stimulations) for each series: 'minimum' (all incompletely documented seizures assumed to be adequate); 'known' (only completely documented seizures rated) and 'maximum' (all incompletely documented seizures assumed to be inadequate).

There were no significant differences between period 1 and period 2 patients for age (means 74.3 and 85.9 ), sex, concurrent treatment with medication with anticonvulsant properties, mean number of treatments ( 7.2 and 8 respectively), incidence of missed seizures ( 4 and $3 \%$ of first stimulations) and global clinical outcome. There were, however, significant differences $(P<0.05,2$ tailed $t$-test) in the minimum, known and maximum inadequate seizure ratings for period 1 and period 2 (means 30 , $47,66 \%$ and $14,14,44 \%$ respectively), failed treatment session ratings (27\% and $17 \%)$ and incidence of partial seizures (19\% and 3\%).

Our findings suggested that two apparently identical E2 machines were not equally effective in inducing adequate seizures. We were aware that there are two possible versions of the E2 (Pippard, 1992) - the unmodified version (E2), which would have a power output at the standard setting used ('ECT $1^{\prime} \times 4$ seconds of $106 \mathrm{mQ}$ ), and the modified version $(\mathrm{E} 2+)$, with an output of $149 \mathrm{mQ}$ at the same setting (both output figures are quoted in units of charge, millicoulombs, and assume a $200 \mathrm{ohm}$ impedance load). The manufacturers confirmed that the inherited machine had been returned for modification in the mid-1980s.

Our audit findings were comparable with Pippard's findings in his audit of ECT in two health regions where an estimated $22 \%$ of applications were considered therapeutically ineffective (Pippard, 1992). Two interrelated factors contribute to the problem: underpowered ECT machines and ignorance on the part of the operator which amplify the problem.

PIPPARD J. (1992) Audit of electro-convulsive treatment in two National Health Service regions. British Journal of Psychiatry, 160, 621-637.

HaKeEm KaZeem, Mossley Hill Hospital, Park Avenue, Liverpool L18 8BU and ToNI Lock, Sefton General Hospital, Smithdown Road, Liverpool L15 $2 \mathrm{HE}$

\section{Mental Health Review Tribunals and the Home Office}

Sir: We much appreciate the comments by Agarwal \& Kumar (Psychiatric Bulletin, 1994. 18. 649-650) about our letter on Mental Health Review Tribunals (Psychiatric Bulletin. 1994, 18, 374).

We do agree with Agarwal \& Kumar that the Home Office passes the buck. Perhaps it does so deliberately.

In considering whether civil servants "hundreds of miles away at the Home Office should ever make decisions about complicated and dangerous patients", Agarwal \& Kumar open up the whole question of whether the system of Home Office control of patients detained under section 41 orders is the best one.

We are aware that these civil servants do not wish to have a psychiatrist among them, preferring to judge questions of parole, transfer and discharge from hospital of restricted patients from the points of view of intelligent and informed lay people. In the current climate of concerned public response to tragedies associated with psychiatric patients in the community, the civil servants are all the more likely to delegate decisions about restricted patients to Mental Health Review Tribunals.

We know too that in Scotland the Mental Welfare Commissioner, who is a psychiatrist, keeps in close contact with the medical officer responsible for restricted patients by visiting him and discussing the relevant issues; in Canada patients detained indefinitely as 'Not Criminally Responsible' are under the jurisdiction of a Provincial Review Board comprising a Judicial Chairman and psychiatric and lay members; and countries in Europe and states in the USA have their own different provisions for governmental control of dangerous mentally abnormal offenders.

Has anybody done worldwide research on procedures in other countries for mentally abnormal patients requiring restrictions?

Chris Green, St Luke's Hospital, Middlesbrough TS4 3AF and GeOFFreY WaLus, Stockton Hall Hospital, York YO3 9UN

\section{Delegation of section 5(2) Mental Health Act 1983 II}

Sir: The issue of who acts as the consultant's nominated deputy continues to crop up. It is a 